\title{
Modeling the thin-layer drying of fruits and vegetables: a review
}

\begin{abstract}
The drying of fruits and vegetables is a complex operation that demands much energy and time. In practice, the drying of fruits and vegetables increases product shelf-life and reduces the bulk and weight of the product, thus simplifying transport. Occasionally, drying may lead to a great decrease in the volume of the product, leading to a decrease in storage space requirements. Studies have shown that dependence purely on experimental drying practices, without mathematical considerations of the drying kinetics, can significantly affect the efficiency of dryers, increase the cost of production, and reduce the quality of the dried product. Thus, the use of mathematical models in estimating the drying kinetics, the behavior, and the energy needed in the drying of agricultural and food products becomes indispensable. This paper presents a comprehensive review of modeling thin-layer drying of fruits and vegetables with particular focus on thin-layer theories, models, and applications since the year 2005. The thin-layer drying behavior of fruits and vegetables is also highlighted. The most frequently used of the newly developed mathematical models for thin-layer drying of fruits and vegetables in the last 10 years are shown. Subsequently, the equations and various conditions used in the estimation of the effective moisture diffusivity, shrinkage effects, and minimum energy requirement are displayed. The authors hope that this review will be of use for future research in terms of modeling, analysis, design, and the optimization of the drying process of fruits and vegetables.
\end{abstract}

Keyword: Diffusion; Drying kinetics; Fruits and vegetables; Mathematical modeling; Thinlayer drying 